\title{
The influence of sex and gonadal hormones on sleep disorders
}

\author{
This article was published in the following Dove Press journal: \\ ChronoPhysiology and Therapy \\ 26 June 2014 \\ Number of times this article has been viewed
}

\author{
Henry J Orff \\ Charles J Meliska \\ L Fernando Martinez \\ Barbara L Parry \\ Department of Psychiatry, University \\ of California, San Diego, CA, USA
}

\begin{abstract}
Sleep disorders such as insomnia, sleep-related breathing disorders, circadian rhythm disorders, and sleep-related movement disorders are a significant public health issue, affecting approximately 40 million people in the US each year. Sleep disturbances are observed in both men and women, though prevalence rates often differ between the sexes. In general, research suggests that women more frequently report subjective complaints of insomnia, yet show better sleep than men when evaluated on objective measures of sleep. Men are more likely to be diagnosed with obstructive sleep apnea than women, though rates of obstructive sleep apnea increase after menopause and may be generally underdiagnosed in women. Although circadian rhythm disorders are equally prevalent in men and women, studies find that women typically have earlier bedtimes and exhibit altered temperature and melatonin rhythms relative to men. Lastly, movement disorders appear to be more prevalent in women than men, presumably due to higher rates of anemia and increased risks associated with pregnancy in women. Although gonadal hormones would be expected to play a significant role in the development and/or exacerbation of sleep disturbances, no causal link between these factors has been clearly established. In large part, the impact of hormones on sleep disturbances is significantly confounded by factors such as psychiatric, physical, and lifestyle concerns, which may play an equal or greater role in the development and/or exacerbation of sleep disturbances than do hormonal factors. Current standard of care for persons with sleep disorders includes use of psychological, pharmacologic, and/or medical device supported interventions. Hormonal-based treatments are not typically recommended given the potential for long-term adverse health effects. In sum, there is a substantial need for more comprehensive studies focused on elucidating the impact of hormones on sleep. Such studies should reveal sex-specific differences in sleep, which could lead to enhanced interventions for sex-specific sleep disturbances.
\end{abstract}

Keywords: sex, sleep disturbance, treatment, prevalence

\section{Introduction to sleep disorders}

Broadly defined, sleep disorders include a wide range of physiological and psychological factors that cause dysregulation in the homeostatic and circadian regulation of sleep leading to impairment in daytime functioning. Sleep disorders include problems of initiating and maintaining sleep, disorders of excessive somnolence, disorders of the sleep-wake schedule, and dysfunctions associated with sleep, sleep stages, or partial arousals (eg, parasomnias). ${ }^{1}$ Poor sleep is a risk factor for chronic diseases (eg, hypertension, diabetes, obesity, and psychiatric illness), puts individuals at increased risk for accidents, and has been shown to decrease quality of life and increase mortality rates in affected individuals. ${ }^{2}$
Correspondence: Henry J Orff UCSD Department of Psychiatry, 9500 Gilman Drive, San Diego, CA 92161 USA

Tel + I 8586426492

Fax +I 8586426340

Email horff@ucsd.edu 
The International Classification of Sleep Disorders- $2^{3}$ differentiates several categories of sleep disorders, of which the most common include insomnia, sleep-related breathing disorders (eg, obstructive sleep apnea [OSA]), circadian rhythm disorders (eg, delayed sleep phase disorder [DSPD]), advanced sleep phase disorder (ASPD), and sleep-related movement disorders (eg, restless legs syndrome [RLS], periodic limb movement disorder [PLMD]). These sleep disorders are notable in that prevalence rates for each differ between men and women. Sleep disorders such as excessive daytime sleepiness (narcolepsy, idiopathic hypersomnia) and parasomnias (eg, sleep-walking, nightmares) tend to show fewer sex differences. Given this observation, disorders of excessive daytime sleepiness will not be addressed directly in this manuscript; however, parasomnias will be discussed in the context of sleep disorders of childhood and adolescence as they represent some of the most common presenting complaints encountered in pediatric settings.

Sleep disorders are a significant public health issue as each year 40 million people in the US suffer from chronic sleep disorders, with another 30 million troubled by transient or occasional sleep problems. ${ }^{4}$ According to the 2002 National Sleep Foundation (NSF) Sleep in America poll, $74 \%$ of respondents reported experiencing at least one symptom of a sleep disorder a few nights per week or more. ${ }^{5}$ The most common complaints reported in the study were insomnia, pauses in breathing, and unpleasant feelings in the legs. Symptoms of insomnia were reported by $58 \%$ of the respondents, with $35 \%$ experiencing symptoms every or almost every night in the past year. Additionally, $9 \%$ of respondents reported pauses in breathing during at least a few nights and $16 \%$ reported symptoms consistent with restless legs syndrome during the past year.

\section{Sex differences and influence of hormones on sleep disorders in children and adolescents}

Sleep disturbances are among the most frequent complaints observed in pediatric practice, with an estimated $25 \%$ of children experiencing some type of sleep problem during childhood and adolescence. ${ }^{6}$ Prevalence rates of parent-reported sleep problems range from $25 \%-50 \%$ in preschool samples ${ }^{7}$ to upwards of $40 \%$ in adolescent samples. ${ }^{8}$ The most commonly reported sleep problems typically involve behavioral issues such as difficulty settling and/or going to bed at night, frequent awakenings at night accompanied with getting out of bed or refusal to return to bed, and difficulty awakening in the morning. Insomnia, as experienced by adults, is less likely to be diagnosed in children and adolescents due to their robust homeostatic drive for sleep. Hence, sleep difficulties in children and adolescents tend to be less related to trouble sleeping per se, but rather manifest from a combination of environmental, behavioral, and medical issues often encountered during this developmental period. Research also suggests that sleep patterns remain relatively stable over time (from age 8-12 years) and do not appear to be significantly affected by pubertal maturity, ${ }^{9}$ nor do there appear to be any significant differences in rates of occurrence of behavioral sleep problems as a function of sex. Although sleep duration may become shorter with age, sleep quality has been found to improve as children enter early adolescence. ${ }^{9}$

Equally common to behavioral sleep problems are the presence of parasomnias in children. Reported prevalence rates for specific parasomnias include sleep talking (55\%), hypnagogic imagery (51\%), sleep starts (33\%), bruxism (28\%), confusional arousals (17\%), night terrors (17\%), movement disorders (17\%), sleepwalking (14\%), sleep paralysis $(7.6 \%)$, and nightmares (5.2\% experiencing one nightmare per week; $10 \%-50 \%$ in children $3-5$ years old). ${ }^{10,11}$ One large study examining parasomnias in children and young adolescents found that enuresis (which was significantly higher in boys) was the only parasomnia that differentiated the sexes. ${ }^{12}$ This study also found that high level of anxiety in children was associated with more frequent experience of night terrors, restless legs, bruxism, and body movement disorders. Generally speaking, parasomnias appear to have a familial component and are observed to decrease with age, though some evidence suggests that sleep talking, restless legs, and bruxism may still be highly prevalent in early teenage years. ${ }^{12}$

Sleep disordered breathing is also a frequently reported concern for children, with prevalence estimates of 3\%-15\% in the general population. ${ }^{13}$ In at-risk obese and overweight children, rates of OSA may be as high $19 \%$ and $41 \%$, respectively. ${ }^{14}$ OSA appears to peak in children during the preschool years when lymphoid tissue of the upper airway is largest in size relative to facial development. OSA, if untreated, can result in significant medical, developmental, and/or psychiatric consequences for children. Research suggests that OSA is significantly associated with obesity and African-American heritage, but shows no association with sex or age. ${ }^{15}$

Circadian rhythm sleep disorders, particularly DSPD, have been well-established as a common problem for adolescents following the onset of puberty. Estimates suggest that between $5 \%$ and $10 \%$ of teens experience DSPD, which 
is thought to occur as a result of both underlying biological factors as well as environmental and lifestyle demands ${ }^{16}$ (for detailed review see Crowley et al). ${ }^{17}$ To date, no specific sex differences in occurrence of this DSPD have been determined.

As opposed to the aforementioned sleep disorders, periodic limb movement disorders appear to be fairly uncommon in children, with prevalence estimates of approximately $1.2 \% .{ }^{18}$ Additionally, few sex differences have been observed in rates of PLMD. ${ }^{19}$ However, PLMD may represent a significant medical concern for children and adolescents, as it appears that having a diagnosis of PLMD is associated with increased risk for attention deficit hyperactivity disorder ${ }^{20,21}$ and greater complications associated with migraine headaches. ${ }^{22}$

\section{Treatment of sleep disorders in children and adolescents}

Generally speaking, treatment of sleep disorders in children and adolescents is similar to recommendations for treatment of sleep disorders in adults. However, given the risk factors associated with use of pharmacologic agents on health and development in children and adolescents, behavioral interventions and/or alternative medical interventions such as acupressure $^{23}$ may be both effective and preferential. Sex-specific and/or hormonal factors are not known to play a major role in onset and/or maintenance of sleep disorders in childhood and adolescence; hence, treatment management strategies should focus on sleep education for parents and children, as well as matching treatment to the child/adolescent's developmental abilities. This is particularly true for difficulties associated with sleep routines, parasomnias, and delayed sleep phase disorder. Use of behavioral contingencies for bedroom and sleep behaviors, relaxation strategies to reduce anxiety, and good sleep hygiene practices are critical to successful intervention and management of sleep disturbances presented in pediatric settings.

\section{Sex differences in qualitative and quantitative sleep in adults}

When comprehensive reports of sleep in normal healthy adults are evaluated, several differences have been typically observed in sleep between sexes. For example, qualitative reports show that women report sleeping longer, but report more disturbed sleep than men. In particular, the National Sleep Foundation Sleep in America poll ${ }^{5}$ found that women reported experiencing symptoms of insomnia at least a few times per week, which included reports of more difficulty falling asleep, waking up more frequently during the night, having more trouble returning to sleep, and feeling less refreshed the following day. Females also reported getting less sleep than preferred on weekdays and were more likely to report daytime sleepiness at least a few times per week. Conversely, males more frequently reported snoring or pauses in breathing at least a few times per week; however, men also reported obtaining more than the minimum hours of sleep needed to not feel sleepy the next day. No apparent differences were noted between males and females with regards to symptoms of restless legs, hours slept on the weekend, or use of prescribed medications for sleep and/or staying awake.

When measured objectively by polysomnography, differences between men and women have also been noted. For example, in a meta-analysis by Ohayon et al, ${ }^{24}$ both sexes showed similar effects of aging on most sleep variables; however, larger effect sizes were observed in women for total sleep time (TST), sleep efficiency, percent stage 1 sleep, and rapid eye movement (REM) latency, suggesting that aging had a greater impact on these variables in women than in men. Also, women appeared to have longer TST and sleep latency, lower percent stage 2 sleep, and greater percentage of slow-wave sleep than age-matched men. Importantly, in addition to age-related changes, women also experience sex-specific physiological changes that potentially disrupt their sleep during the menstrual cycle,${ }^{25}$ pregnancy,${ }^{26}$ in the postpartum period, ${ }^{27}$ and at menopause. ${ }^{28}$

\section{Sex differences and influence of hormones on sleep disorders in adults \\ Insomnia}

Insomnia affects approximately $10 \%-15 \%$ of adults in the US, with as many as $30 \%$ of individuals reporting poor sleep quality. ${ }^{29}$ Differences between sexes in reports of insomnia have also been observed, with survey estimates suggesting that $27 \%$ of women versus $19 \%$ of men have an insomnia complaint. ${ }^{30}$ Additionally, women are more likely to report sleep disturbances and are $41 \%$ more likely than men to experience insomnia. ${ }^{31}$

To date, the etiology of these self-reported sex differences in insomnia is not well defined. In women, insomnia may result from multifactorial issues, including hormonal changes associated with the menstrual cycle, pregnancy, postpartum, hot flashes associated with menopause, oral contraceptive use, psychiatric complaints (eg, anxiety and depression), undiagnosed sleep disorders such as apnea, 
and/or lifestyle and environmental factors. In particular, hormonal changes during the late-luteal phase of the menstrual cycle, third trimester of pregnancy, and menopause are strongly associated with women's reports of insomnia. ${ }^{32,33}$

With regards to the menstrual cycle, evidence for hormonal influences on sleep has been sparse and inconclusive. Research on sleep during the menstrual cycle in healthy women has failed to show significant variation across the menstrual cycle in objective ratings of major sleep variables including TST, sleep efficiency, sleep latency, REM sleep latency, or slow wave sleep; ${ }^{34}$ however, Shechter et al found evidence for decreased REM and increased stage 2 and slow-wave sleep during the luteal compared with the follicular phase. ${ }^{35}$ In women with premenstrual syndrome (PMS) or premenstrual dysphoric disorder (PMDD), quantitative polysomnographic studies have shown that women with severe PMS experience more awakenings in the late-luteal phase compared with the follicular phase, though similar findings have been observed in asymptomatic women. ${ }^{36}$ Compared with healthy women, those with PMS/PMDD show evidence of decreased delta and increased theta activity and amplitude (regardless of menstrual phase), indicative of possible electroencephalographic trait markers of PMS/PMDD. Parry et al also found that women with PMDD displayed increased stage 2 sleep and less REM sleep (irrespective of menstrual phase), and less stage 3 sleep and fewer awakenings during the lateluteal phase when compared with healthy women. ${ }^{37}$ These limited findings of disruption in objective sleep also appear to extend to women with dysmenorrhea and women using oral contraceptives. ${ }^{38}$ Hence, the role of hormonal influence on sleep during the menstrual cycle is not well understood and has not shown consistent findings.

Pregnancy is also a time of hormonal, physical, and emotional changes that can disrupt sleep. Studies consistently show that sleep disturbance increases as pregnancy progresses, becoming particularly problematic in the third trimester. ${ }^{39,40}$ In general, women primarily experience fatigue during the first trimester, less disruption of sleep during the second trimester, and more significant generalized disruption of sleep during the third trimester and postpartum. ${ }^{38}$ When evaluated objectively, studies of sleep in pregnant women typically have yielded equivocal results. For example, in a study of women in the third trimester, awakenings and stage 1 sleep were found to increase while sleep efficiency and REM percent decreased compared with nonpregnant women. ${ }^{41}$ Other studies show a trend towards increased awakenings from the second to third trimesters and decreased REM sleep from the first to second trimesters. ${ }^{42}$ Additionally, women appear to experience decreased slow-wave sleep, ${ }^{43-46}$ but increased total sleep times, ${ }^{47}$ as pregnancy progresses. However, one study ${ }^{48}$ showed that slow-wave sleep was significantly greater at 27-39 weeks than at 8-16 weeks, while REM sleep remained unchanged. Studies evaluating subjective measures of sleep indicate pregnant women report sleeping roughly the same amount as nonpregnant women per night, although it may be the case that pregnant women alter their sleep patterns to compensate for nighttime awakenings by going to bed earlier, napping, and sleeping later when possible. Therefore, while hormonal changes likely play some role in sleep disruption during pregnancy, their influence may be limited, especially when compared with factors such as physical discomfort, leg cramps, nocturia, and/or pain that occur during pregnancy and that may lead to disruption of sleep.

During postpartum, sleep in women can be significantly disrupted. This disruption is likely due to a multitude of factors. As with pregnancy, however, few comprehensive objective studies of postpartum sleep have been published. One objective sleep study found that the most significant effects on sleep occur at 4 weeks postpartum, at which time sleep continuity becomes disrupted due to wakefulness (1 hour per night) associated with infant care. ${ }^{49}$ Other studies typically show normalization to pre-pregnant levels of sleep efficiency and nighttime awakenings by 3-5 months postpartum. Subjective and objective studies conducted on new mothers and their infants show that by around three months, the infants' sleep and wake patterns become more regular and, in turn, the mothers' sleep becomes more continuous. ${ }^{50,51}$ Thus, as in pregnant women, it appears that sleep disruption in postpartum women may be equally or more significantly related to parenting demands as opposed to hormonal changes per se.

Increased sleep disruptions are common complaints in women during perimenopause and menopause. In fact, as many as $50 \%$ of women subjectively report significant sleep complaints during this time. ${ }^{52}$ When objective sleep in perimenopausal and menopausal women is evaluated, however, results appear less conclusive. For example, in one study of menopausal women, those women who were described as poor sleepers showed more wakefulness and stage 2 sleep, and less REM sleep, than those women described as good sleepers. ${ }^{53}$ Additionally, pre- and postmenopausal women have less slow-wave sleep and higher frequency and duration of awakenings than younger women. ${ }^{54}$ This latter study also showed that, while total sleep times were similar in premenopausal and postmenopausal 
women, total sleep times were longer and sleep efficiencies greater in younger women as compared with premenopausal and postmenopausal women. ${ }^{54}$ Conversely, in a large epidemiologic study, ${ }^{55}$ objective sleep quality was not worse in peri- or postmenopausal women than in premenopausal women, with postmenopausal woman actually exhibiting more deep sleep and significantly longer total sleep times. With regard to the influence of hormones on sleep during this period of time, decreases in both estrogen and progesterone have been implicated as possible causal factors in the observed sleep alterations. These changes, particularly for progesterone, which has sleep protective properties, may contribute to difficulties falling asleep and to greater susceptibility to environmental and psychological factors that can disrupt sleep. Lower levels of estrogen are also associated with increased mood symptoms that can occur in about $20 \%$ of women at this time and which also may be associated with poor sleep. Also, increased adrenal activity during this time is associated with the development of hot flashes, which can disrupt sleep significantly. ${ }^{56}$ Therefore, unlike other periods of significant hormonal change in the female reproductive lifecycle, there is more evidence supporting the role of hormones on sleep disturbance during the perimenopausal and menopausal period.

It should be noted that there is limited but important evidence to suggest that steroidal hormones may impact sleep in men. Abnormalities of sleep quality, duration, circadian rhythm disruption, and sleep-disordered breathing may reduce testosterone levels. ${ }^{57}$ In a study of men over 65 years of age, lower testosterone levels were associated with reduced sleep efficiency, increased nocturnal awakenings, and less slow-wave sleep. ${ }^{58}$ Conversely, testosterone administration and heavy androgenic/anabolic steroid use is associated with reduced sleep times, increased number of awakenings, and more frequent reports of insomnia. ${ }^{59}$

\section{Sleep-related breathing disorders}

Sleep apnea is a common sleep disorder characterized by pauses in breathing or instances of shallow or infrequent breathing during sleep. OSA, the most common form of apnea, is diagnosed in approximately $84 \%$ of apnea-related cases. By comparison, central sleep apnea and mixed or complex sleep apnea are only diagnosed in $15 \%$ and $0.4 \%$ of cases, respectively. According to the Wisconsin Sleep Cohort Study, one in 15 people in the US may be affected by at least moderate levels of sleep apnea, ${ }^{60,61}$ with as much as $9 \%$ of middle-aged women and $24 \%$ of middle-aged men affected, undiagnosed, and untreated. ${ }^{61,62}$
Misdiagnosis of OSA in women is thought to be common, due in large part to the fact that women often present with atypical apnea symptoms, such as chronic fatigue, insomnia, morning headaches, and mood disturbance, as opposed to the classic symptoms of OSA, such as snoring, witnessed pauses in breathing at night, and excessive sleepiness during the day, which typify complaints among men. In particular, women are at risk for developing OSA or exacerbation of OSA symptoms during pregnancy due primarily to physical and possibly hormonal changes that occur during this time. ${ }^{63}$ This finding is of great importance as studies show that women with severe OSA also have a higher incidence rate of adverse pregnancy outcomes, driven in large part by increased prevalence of gestational diabetes and preterm births. ${ }^{64}$

With regards to menopause, the data supporting increased OSA levels are less clear. In the Wisconsin Sleep Cohort study, ${ }^{65}$ investigators found that mild sleep apnea (apnea/hypopnea index $<10$ ) was found in $16 \%$ of women 50-60 years of age; however, OSA was not associated with increasing age. One of the primary hypothesized causes for increased OSA severity in postmenopausal women relates to increased body fat distribution (particularly increased waste-to-hip ratio), as well as hormonal changes (especially decreased progesterone production). While changes in body fat composition are an important consideration, one large cross-sectional polysomnographic study ${ }^{66}$ determined that prevalence and severity of sleep-disordered breathing was found to increase in postmenopausal versus premenopausal women even after controlling for body mass index and neck circumference. As progesterone is a known respiratory stimulant in women, increases in OSA during the postmenopausal period might be influenced by changes in levels of this hormone. Nevertheless, progesterone administration may not be effective in the treatment of OSA in either men ${ }^{67}$ or women. ${ }^{68}$

Despite men exhibiting higher levels of OSA than women, little is known about the effects of steroidal hormones in men and their effect on sleep disordered breathing. Most of the evidence relating OSA to changes in testosterone is correlational. For example, changes in testosterone levels occur during sleep in both men and women, with testosterone levels rising during sleep (highest during REM sleep) and decreasing during waking hours. When an individual suffers from a sleep disorder such as OSA, sleep becomes fragmented, and overall sleep and REM sleep levels become reduced, hence leading to lower testosterone levels. Studies have shown that low testosterone levels frequently occur in men with OSA, ${ }^{58}$ and men with OSA are also more 
likely to suffer from complications in sexual functioning, including low libido, erectile dysfunction, and impotence. ${ }^{69,70}$ In a treatment study, successful treatment with continuous positive airway pressure (CPAP) therapy resulted in a partial increase of pituitary/gonadal function with mild increases in luteinizing hormone and testosterone secretory characteristics. ${ }^{71}$ In this study, however, men treated for OSA still exhibited lower luteinizing hormone and testosterone levels than men in the control group. Hence, the relationship between steroidal hormones and OSA remains unclear.

\section{Circadian rhythm related sleep disorders}

Circadian rhythm related sleep disorders are caused by a mismatch between the timing of sleep and peak periods of alertness with the requirements of an individual's daily life responsibilities. Circadian rhythm sleep disorders include DSPD, ASPD, and non-24-hour (free-running type) sleepwake disorder, as well as problems related to jet lag and shift work. DSPD is the most common circadian rhythm disorder and results in a preferred sleep phase that may be delayed until after midnight and lasting until late morning. ASPD, which is more common in elderly patients, results in an advanced sleep phase such that individuals fall asleep in the early evening and wake in the early morning hours. Non-24-hour sleep-wake disorder causes an individual to fall asleep approximately 1 to 2 hours later each consecutive day (hence corresponding to a circadian cycle of 25-26 hours). DSPD appears to be equally common in men and women; however, one large survey of morningness-eveningness preference in university students showed that men have a significantly greater eveningness preference compared with women. ${ }^{72}$ ASPD affects men and women equally and has a strong genetic link, with $40 \%-50 \%$ of patients having a relative with ASPD. Non-24-hour sleep disorder is rare and most commonly observed in individuals who are totally blind, though fully-sighted individuals can be affected as well. Currently, there is insufficient data to assess whether sex is a risk factor for non-24-hour sleep disorder. ${ }^{73}$

The evidence for hormonal involvement in circadian rhythm disorders is based to a large degree on differences noted in male and female circadian biology. For example, sex differences observed in the nervous system that underlie circadian functioning result in large part from developmental exposure to hormones that help differentiate and organize functional differences between the sexes during the prenatal and adolescent periods. ${ }^{74}$ Second, as noted previously, men and women sleep differently. Women spend more time in bed and sleep longer, but report a poorer sleep quality than men. ${ }^{75}$ Also, women tend to go to bed earlier than men from childhood to menopause, after which sex differences disappear. ${ }^{76}$ Third, studies show that women exhibit phaseadvanced rhythms (eg, temperature and melatonin) such that circadian timing is shifted earlier in women than in men; ${ }^{77,78}$ hence, women tend to sleep longer than men. ${ }^{79}$ Fourth, the fact that women experience fluctuating levels of hormones throughout their reproductive lifespan has been proposed as a mechanism by which sleep and circadian disorders may occur more frequently in women than men. ${ }^{80,81}$ Lastly, across both sexes, hormonal rhythms appear to be significantly delayed in patients with DSPD, ${ }^{82}$ and such circadian misalignments may adversely affect metabolic and hormonal factors such as circulating glucose and insulin levels. ${ }^{83}$

\section{Sleep-related movement disorders}

RLS is a neurological disorder characterized by unpleasant sensations in the legs, which occur primarily at night (when a person is relaxing or at rest) and which are typically relieved through movement. Approximately $10 \%$ of persons in the US may have RLS, with $2 \%-3 \%$ experiencing moderate to severe RLS symptoms. ${ }^{84}$ More than $80 \%$ of people with RLS also experience a condition known as PLMD. PLMD is characterized by involuntary leg twitching or jerking movements during sleep that typically occur every 15 to 40 seconds, sometimes throughout the night. The symptoms cause repeated awakening and severely disrupted sleep. Although many individuals with RLS also develop PLMD, most people with PLMD do not experience RLS. RLS occurs in both men and women, although the incidence is about twice as high in women. ${ }^{85}$

In most cases, the cause of RLS is unknown; however, RLS appears to have a genetic component as family history is typically positive for individuals whose onset of symptoms is before the age of 40 years. The putative causes for RLS include low levels of iron and/or dysfunction in basal ganglia circuits that use the neurotransmitter dopamine. RLS may also be related to chronic conditions such as kidney failure, diabetes, and peripheral neuropathy. For women, pregnancy, especially in the last trimester, has been associated with increased risk for RLS that typically disappears within 4 weeks following delivery. In particular, one cohort study of women studied during and after pregnancy showed that $26 \%$ of women reported RLS during pregnancy (most common during the third trimester) that tended to disappear after delivery. ${ }^{86}$ Women who reported RLS symptoms in this study also exhibited lower hemoglobin concentrations compared with nonaffected women, a finding supporting 
the hypothesis that iron deficiency contributes to RLS. Additionally, thyroid stimulating hormone has been implicated in RLS as conditions associated with higher levels of thyroid hormones (such as pregnancy) have higher prevalence of RLS symptoms. Beyond these factors, there is no evidence to suggest that hormones play a specific role in onset and/or exacerbation of RLS symptoms.

\section{Treatment of sleep disorders in adults Insomnia}

The most common treatment for insomnia symptoms is prescription and over-the-counter sedative hypnotic medications. According to recent statistics, approximately $4 \%$ of US adults aged 20 years and older use prescription drugs for sleep on a regular basis, with rates of medication use higher among women than men (5\% versus 3.1\%). ${ }^{87}$ US Food and Drug Administration approved treatments for insomnia include benzodiazepine receptor agonists and melatonin receptor agonists. Most sleep medications produce negative side effects including dependence, sedation, cognitive impairment, risk for falls, and possible increased risk for parasomnias. Long-term use of hypnotics also appears to be associated with increased rates of cancer and morbidity. ${ }^{88}$ As comorbid depression and insomnia are more prevalent in women than in men, selective serotonin reuptake inhibitors may also be an effective alternative treatment of sleep disturbances. ${ }^{89}$ Other medications including over-the-counter antihistamines and herbal supplements such as melatonin and valerian are used to treat insomnia, although safety and efficacy data are limited for these treatments..$^{90}$

As an alternative to hypnotic medications, cognitivebehavioral therapy for insomnia (CBT-I) is commonly recommended for the treatment of sleep initiation and maintenance problems. In fact, the American Academy of Sleep Medicine now recommends CBT-I as the first-line treatment for chronic insomnia. ${ }^{91}$ Empirical data show CBT-I to be equally effective in the short-term treatment of insomnia and superior in the long-term management of insomnia compared to pharmacological treatment. ${ }^{92-94}$ Standard psychologicalbased treatments for insomnia include cognitive and/or behavioral strategies, sleep hygiene education, stimulus control therapy, sleep restriction therapy, relaxation, and/or biofeedback.

Hormone replacement therapy (HRT) has also been proposed as an alternative treatment for insomnia. In particular, estrogen supplementation may reduce hot flashes in menopausal women, thereby reducing the deleterious effects of hot flashes on sleep. Typically HRT is recommended for the shortest possible term and in the lowest possible dose given the increased risk for breast cancer and cardiovascular conditions, particularly in older postmenopausal women. Alternatively, some women may also benefit from use of a low-dose birth control pill, which may help stabilize mild fluctuations of estrogen. Research suggests, however, that use of exogenous hormones may negatively impact sleep by raising body temperature and altering sleep (producing greater stage 2 sleep and less slow-wave sleep), an effect not observed to occur from endogenous progesterone and estrogen produced in naturally cycling women. ${ }^{95}$

\section{Sleep-related breathing disorders}

The most common approach to treating sleep apnea is use of assisted breathing and or dental devices that help maintain an open airway during sleep. CPAP therapy has been shown to be effective in reducing OSA symptoms, though compliance with this form of treatment is typically low. At this time, the impact of sex on acceptance, adherence, and long-term treatment outcomes is largely unexplored and unknown, given that current studies are confounded by factors such as age, disease severity, and symptomatic severity. ${ }^{96}$ Alternatives to CPAP include: use of oral appliances (mouthpieces); nasal sprays or breathing strips; behavioral lifestyle-based changes such as adjusting sleep position to reduce snoring; losing weight; quitting smoking; and avoiding alcohol and medications that increase sleepiness. ${ }^{97}$ In very severe cases of apnea, surgery also may be an option to open breathing passages (eg, removing tonsils) or to reduce weight (eg, bariatric surgery). To date, most of the treatment studies conducted on apnea patients have been conducted in men; hence, the role of non-CPAP therapies has not been adequately assessed in women and/or has yielded conflicting results. ${ }^{98}$ However, some studies indicate that hormone therapy may reduce apnea symptoms. ${ }^{99,100}$ Despite these findings, the Standards of Practice Committee of the American Academy of Sleep Medicine ${ }^{101}$ found no obvious benefit for use of HRT for OSA in a survey of the literature, and, given the high risks for adverse side effects associated with HRT, recommended against the use of HRT specifically for treatment of OSA in postmenopausal women.

\section{Circadian rhythm disorders}

Treatment of circadian rhythm disorders is similar for both sexes and includes a combination of adjusting sleep schedules, critically timed bright light exposure, and use 
of melatonin. Behaviorally, circadian rhythm disorders can be treated through gradually delaying or advancing an individual's sleep schedule by 2-3 hours per day for 1 week until the desired sleep schedule is achieved. Circadian rhythm disorders also respond well to bright light exposure therapy. For individuals with a delayed sleep schedule, morning bright light for 30-60 minutes ( $>6,000 \mathrm{lux})$ is often effective in advancing sleep onset times. Conversely, evening light can help delay sleep onset in those with advanced sleep phase syndrome. Incorporating exogenous melatonin (0.3-3 mg taken in the evening) has been found to help advance sleep phase in those with delayed sleep phase disorder, ${ }^{102}$ especially when combined with light therapy. ${ }^{103}$ Finally, other factors such as improved sleep hygiene and making lifestyle changes (especially for shift workers) can be helpful in reducing the effects of circadian rhythm disorders. With the exception of melatonin, there are no currently recommended or prescribed hormonal therapies for circadian rhythm disorders.

\section{Movement disorders}

Although the underlying cause of RLS/PLMD is not fully understood, dopamine dysregulation is suspected to be the primary cause. ${ }^{104-106}$ Recommended treatments for RLS therefore primarily include pharmacologic administration of dopamine agonists (pramipexole, ropinirole). Also, iron supplementation is often recommended in patients with low iron levels. ${ }^{107}$ Behavioral strategies such as exercise therapy have not been well tested. ${ }^{87}$ In general, patients are encouraged to avoid caffeine, alcohol, and medications such as antihistamines, neuroleptics, dopamine antagonists, certain selective serotonin reuptake inhibitors, tricyclic antidepressants, and lithium, all of which may exacerbate RLS symptoms. No sex-related differences in treatment outcomes for RLS have been observed, ${ }^{108}$ hence, hormonal-based treatments for RLS are not currently recommended.

\section{Conclusion and therapy implications}

In summary, sleep disturbances are common to both men and women, and sex differences exist for certain sleep disorders. While women appear to obtain more sleep than men, they also tend to report more frequent symptoms of insomnia. On the other hand, men tend to be more frequently diagnosed with sleep disordered breathing than women, although apnea may be underdiagnosed in women. Despite the presumed importance hormones may have for sleep maintenance and disruption, a clear influence of hormones on sleep has yet to be established. However, research evidence supports a role for hormones in sleep disruption, especially for women during menopause. In most cases, sleep disruption in men and women is confounded by psychiatric, physical, and lifestyle factors that may play an equal or greater role in development of sleep disturbances than those exerted by hormonal factors. Although a complete clinical workup for sleep disturbance should include queries regarding hormonal concerns, the current standard of care for persons with sleep disorders primarily includes cognitive behavioral, pharmacologic, and/or medical device supported interventions. Use of hormonal replacement therapies is typically not recommended as a first-line intervention for sleep disruptions given the potential long-term risks for adverse health effects. In sum, there is a critical need for comprehensive studies focused on elucidating the impact of hormones on sleep. Such studies should reveal sex-specific differences in sleep, which could lead to interventions for sex-specific sleep disturbances.

\section{Disclosure}

The authors have no conflicts of interest in this work.

\section{References}

1. Cormier RE. Sleep Disturbances. In: Walker HK, Hall WD, Hurst JW, editors. Clinical Methods: The History, Physical, and Laboratory Examinations. 3rd ed. Boston: Butterworths; 1990. Available at http://www. ncbi.nlm.nih.gov/books/NBK401/. Accessed June 10, 2014.

2. Functional and economic impact of sleep loss and sleep-related disorders. In: Institute of Medicine (US) Committee on Sleep Medicine and Research; Colten HR, Altevogt BM, editors. Sleep Disorders and Sleep Deprivation: An Unmet Public Health Problem. Washington, DC: National Academies Press (US); 2006. Available at http://www.ncbi. nlm.nih.gov/books/NBK19958/. Accessed June 10, 2014.

3. American Academy of Sleep Medicine. International Classification of Sleep Disorders - Second Edition (ICSD-2), Westchester, IL: American Academy of Sleep Medicine; 2005.

4. Brain Basics: Understanding Sleep [webpage on the Internet]. Bethesda: National Institute of Neurological Disorders and Stroke; 2013; Available from: http://www.ninds.nih.gov/disorders/brain_basics/ understanding_sleep.htm\#sleep_disorders. Accessed April 1, 2014.

5. National Sleep Foundation 2012 Bedroom Poll: Summary of Findings [webpage on the Internet]. Arlington, VA: National Sleep Foundation; 2012; Available from http://sleepfoundation.org/sleep-polls-data/otherpolls/2012-bedroom-poll. Accessed April 1, 2014.

6. Mindell JA, Owens JA, Carskadon MA. Developmental features of sleep. Child Adolesc Psychiatr Clin N Am. 1999;8(4):695-725.

7. Kerr S, Jowett S. Sleep problems in pre-school children: a review of the literature. Child Care Health Dev. 1994;20(6):379-391.

8. Giannotti F, Cortesi F. Sleep patterns and daytime functions in adolescents: an epidemiological survey of Italian high-school student population. In: Carskadon MA, editor. Adolescent Sleep Patterns: Biological, Social, and Psychological Influences. New York: Cambridge University Press; 2002:132-147.

9. Pesonen AK, Martikainen S, Heinonen K, et al. Continuity and change in poor sleep from childhood to early adolescence. Sleep. 2014;37(2): 289-297.

10. Waters KA, Suresh S, Nixon GM. Sleep disorders in children. Med J Aust. 2013;199(8):S31-S35.

11. Moore M, Allison D, Rosen CL. A review of pediatric nonrespiratory sleep disorders. Chest. 2006;130(4):1252-1262. 
12. Laberge L, Tremblay RE, Vitaro F, Montplaisir J. Development of parasomnias from childhood to early adolescence. Pediatrics. 2000; 106(1 Pt 1):67-74.

13. Raynes-Greenow CH, Hadfield RM, Cistulli PA, Bowen J, Allen H, Roberts CL. Sleep apnea in early childhood associated with preterm birth but not small for gestational age: a population-based record linkage study. Sleep. 2012;35(11):1475-1480.

14. Verhulst SL, Schrauwen N, Haentjens D, et al. Sleep-disordered breathing in overweight and obese children and adolescents: prevalence, characteristics and the role of fat distribution. Arch Dis Child. 2007;92(3):205-208

15. Redline S, Tishler PV, Schluchter M, Aylor J, Clark K, Graham G. Risk factors for sleep-disordered breathing in children. Associations with obesity, race, and respiratory problems. Am J Respir Crit Care Med. 1999;159(5 Pt 1):1527-1532.

16. Carskadon MA, Vieira C, Acebo, C. Association between puberty and delayed phase preference. Sleep. 1993;16(3):258-262.

17. Crowley SJ, Acebo C, Carskadon MA. Sleep, circadian rhythms, and delayed phase in adolescence. Sleep Med. 2007;8(6):602-612.

18. Kirk VG, Bohn S. Periodic limb movements in children: prevalence in a referred population. Sleep. 2004;27(2):313-315.

19. Picchietti D, Allen RP, Walters AS, Davidson JE, Myers A, FeriniStrambi L. Restless legs syndrome: prevalence and impact in children and adolescents - the Peds REST study. Pediatrics. 2007;120(2) 253-266.

20. Picchietti DL, Walters AS. Moderate to severe periodic limb movement disorder in childhood and adolescence. Sleep. 1999;22(3):297-300.

21. Miano S, Donfrancesco R, Parisi P, et al. Case reports of sleep phenotypes of ADHD: from hypothesis to clinical practice. J Atten Disord. 2013;17(7):565-573.

22. Esposito M, Parisi P, Miano S, Carotenuto M. Migraine and periodic limb movement disorders in sleep in children: a preliminary case-control study. J Headache Pain. 2013;14:57.

23. Carotenuto M, Gallai B, Parisi L, Roccella M, Esposito M. Acupressure therapy for insomnia in adolescents: a polysomnographic study. Neuropsychiatr Dis Treat. 2013;9:157-162.

24. Ohayon MM, Carskadon MA, Guilleminault C, Vitiello MV. Meta-analysis of quantitative sleep parameters from childhood to old age in healthy individuals: developing normative sleep values across the human lifespan. Sleep. 2004;27(7):1255-1273.

25. Hachul H, Andersen ML, Bittencourt LR, Santos-Silva R, Conway SG, Tufik S. Does the reproductive cycle influence sleep patterns in women with sleep complaints? Climacteric. 2010;13(6):594-603.

26. Wilson DL, Barnes M, Ellett L, Permezel M, Jackson M, Crowe SF. Decreased sleep efficiency, increased wake after sleep onset and increased cortical arousals in late pregnancy. Aust $N \mathrm{Z}$ Obstet Gynaecol. 2011;51(1):38-46.

27. Insana SP, Stacom EE, Montgomery-Downs HE. Actual and perceived sleep: associations with daytime functioning among postpartum women. Physiol Behav. 2011;102(2):234-238.

28. Pien GW, Sammel MD, Freeman EW, Lin H, DeBlasis TL. Predictors of sleep quality in women in the menopausal transition. Sleep. 2008;31(7):991-999.

29. Ohayon MM. Epidemiology of insomnia: what we know and what we still need to learn. Sleep Med Rev. 2002;6(2):97-111.

30. Kessler RC, Berglund PA, Coulouvrat C, et al. Insomnia and the performance of US workers: results from the America insomnia survey. Sleep. 2011;34(9):1161-1171.

31. Zhang B, Wing YK. Sex differences in insomnia: a meta-analysis. Sleep. 2006;29(1):85-93.

32. Krystal AD. Insomnia in women. Clin Cornerstone. 2003;5(3):41-50.

33. Krystal AD, Edinger J, Wohlgemuth W, Marsh GR. Sleep in perimenopausal and post-menopausal women. Sleep Med Rev. 1998;2(4): 243-253.

34. Driver HS, Dijk DJ, Werth E, Biedermann K, Borbely AA. Sleep and the sleep electroencephalogram across the menstrual cycle in young healthy women. J Clin Endocrinol Metab. 1996;81(2):728-735.
35. Shechter A, Varin F, Boivin DB. Circadian variation of sleep during the follicular and luteal phases of the menstrual cycle. Sleep. 2010;33(5):647-656.

36. Baker FC, Kahan TL, Trinder J, Colrain IM. Sleep quality and the sleep electroencephalogram in women with severe premenstrual syndrome. Sleep. 2007;30(10):1283-1291.

37. Parry BL, Mendelson WB, Duncan WC, Sack DA, Wehr TA. Longitudinal sleep EEG, temperature, and activity measurements across the menstrual cycle in patients with premenstrual depression and in age-matched controls. Psychiatry Res. 1989;30(3):285-303.

38. Moline ML, Broch L, Zak R, Gross V. Sleep in women across the life cycle from adulthood through menopause. Sleep Med Rev. 2003;7(2): 155-177.

39. Facco FL, Kramer J, Ho KH, Zee PC, Grobman WA. Sleep disturbances in pregnancy. Obstet Gynecol. 2010;115(1):77-83.

40. Kizilirmak A, Timur S, Kartal B. Insomnia in pregnancy and factors related to insomnia. ScientificWorldJournal. 2012;2012:197093.

41. Hertz G, Fast A, Feinsilver SH, Albertario CL, Schulman H, Fein AM. Sleep in normal late pregnancy. Sleep. 1992;15(3):246-251.

42. Brunner DP, Munch M, Biedermann K, Huch R, Huch A, Borbely AA. Changes in sleep and sleep electroencephalogram during pregnancy. Sleep. 1994;17(7):576-582.

43. Schorr SJ, Chawla A, Devidas M, Sullivan CA, Naef RW 3rd, Morrison JC Sleep patterns in pregnancy: a longitudinal study of polysomnography recordings during pregnancy. J Perinatol. 1998;18(6 Pt 1):427-430.

44. Petre-Quadens O, De Lee C. Sleep-cycle alterations during pregnancy, postpartum, and the menstrual cycle. In: Ferin M, Halberg F, Richart R, Van de Wiele RL, editors. Biorhythms and Human Reproduction. New York: John Wiley and Sons; 1974:335-352.

45. Roffwarg HP, Frankel BL, Pessah M, editors. The nocturnal sleep pattern in pregnancy. Annual Meeting of the Association for the Psychophysiological Study of Sleep; Mar 1968; Denver, CO.

46. Karacan I, Williams WB, Webb H, Agnew RJ. Abstracts. Association for the Psychophysiology Study of Sleep Meeting. 1967;4:378; Santa Monica, CA.

47. Lee KA, Zaffke ME, McEnany G. Parity and sleep patterns during and after pregnancy. Obstet Gynecol. 2000;95(1):14-18.

48. Driver HS, Shapiro CM. A longitudinal study of sleep stages in young women during pregnancy and postpartum. Sleep. 1992;15(5): 449-453.

49. Coble PA, Reynolds CF 3rd, Kupfer DJ, Houck PR, Day NL, Giles DE. Childbearing in women with and without a history of affective disorder. II. Electroencephalographic sleep. Compr Psychiatry. 1994;35(3):215-224.

50. Shinkoda H, Matsumoto K, Park YM. Changes in sleep-wake cycle during the period from late pregnancy to puerperium identified through the wrist actigraph and sleep logs. Psychiatry Clin Neurosci. 1999;53(2):133-135.

51. Horiuchi S, Nishihara K. Analyses of mothers' sleep logs in postpartum periods. Psychiatry Clin Neurosci. 1999;53(2):137-139.

52. Hachul H, Bittencourt LR, Soares JM Jr, Tufik S, Baracat EC. Sleep in post-menopausal women: differences between early and late postmenopause. Eur J Obstet Gynecol Reprod Biol. 2009;145(1):81-84.

53. Shaver JL, Giblin E, Paulsen V. Sleep quality subtypes in midlife women. Sleep. 1991;14(1):18-23.

54. Kalleinen N, Polo-Kantola P, Himanen SL, et al. Sleep and the menopause - do postmenopausal women experience worse sleep than premenopausal women? Menopause Int. 2008;14(3):97-104.

55. Young T, Rabago D, Zgierska A, Austin D, Laurel F. Objective and subjective sleep quality in premenopausal, perimenopausal, and postmenopausal women in the Wisconsin Sleep Cohort Study. Sleep. 2003;26(6):667-672.

56. Meldrum DR, Tataryn IV, Frumar AM, Erlik Y, Lu KH, Judd HL. Gonadotropins, estrogens, and adrenal steroids during the menopausal hot flash. J Clin Endocrinol Metab. 1980;50(4):685-689.

57. Wittert G. The relationship between sleep disorders and testosterone in men. Asian J Androl. January 7, 2014. 
58. Barrett-Connor E, Dam TT, Stone K, Harrison SL, Redline S, Orwoll E. The association of testosterone levels with overall sleep quality, sleep architecture, and sleep-disordered breathing. J Clin Endocrinol Metab. 2008;93(7):2602-2609.

59. Liu PY, Yee B, Wishart SM, et al. The short-term effects of high-dose testosterone on sleep, breathing, and function in older men. $J$ Clin Endocrinol Metab. 2003;88(8):3605-3613.

60. Young T, Palta M, Dempsey J, Skatrud J, Weber S, Badr S. The occurrence of sleep-disordered breathing among middle-aged adults. N Engl J Med. 1993;328(17):1230-1235.

61. Lee W, Nagubadi S, Kryger MH, Mokhlesi B. Epidemiology of obstructive sleep apnea: a population-based perspective. Expert Rev Respir Med. 2008;2(3):349-364.

62. Young T, Peppard PE, Gottlieb DJ. Epidemiology of obstructive sleep apnea: a population health perspective. Am J Respir Crit Care Med. 2002;165(9):1217-1239.

63. Venkata C, Venkateshiah SB. Sleep-disordered breathing during pregnancy. J Am Board Fam Med. 2009;22(2):158-168.

64. Facco FL, Liu CS, Cabello AA, Kick A, Grobman WA, Zee PC. Sleep-disordered breathing: a risk factor for adverse pregnancy outcomes? Am J Perinatol. 2012;29(4):277-282.

65. Young T. Rationale, design, and findings from the Wisconsin sleep Cohort study: toward understanding the total societal burden of sleepdisordered breathing. Sleep Med Clin. 2009;4(1):37-46.

66. Dancey DR, Hanly PJ, Soong C, Lee B, Hoffstein V. Impact of menopause on the prevalence and severity of sleep apnea. Chest. 2001;120(1):151-155.

67. Rajagopal KR, Abbrecht PH, Jabbari B. Effects of medroxyprogesterone acetate in obstructive sleep apnea. Chest. 1986;90(6):815-821.

68. Cistulli PA, Barnes DJ, Grunstein RR, Sullivan CE. Effect of short-term hormone replacement in the treatment of obstructive sleep apnoea in postmenopausal women. Thorax. 1994;49(7):699-702.

69. Karacan I, Karatas M. Erectile dysfunction in sleep apnea and response to CPAP. J Sex Marital Ther. 1995;21(4):239-247.

70. Budweiser S, Enderlein S, Jörres RA, et al. Sleep apnea is an independent correlate of erectile and sexual dysfunction. $J$ Sex Med. 2009;6(11): 3147-3157.

71. Luboshitzky R, Aviv A, Hefetz A, et al. Decreased pituitary-gonadal secretion in men with obstructive sleep apnea. J Clin Endocrinol Metab. 2002;87(7):3394-3398.

72. Adan A, Natale V. Gender differences in morningness-eveningness preference. Chronobiol Int. 2002;19(4):709-720.

73. Sack RL, Auckley D, Auger RR, et al. Circadian rhythm sleep disorders: part II, advanced sleep phase disorder, delayed sleep phase disorder, free-running disorder, and irregular sleep-wake rhythm. An American Academy of Sleep Medicine review. Sleep. 2007;30(11):1484-1501.

74. Mong JA, Baker FC, Mahoney MM, et al. Sleep, rhythms, and the endocrine brain: influence of sex and gonadal hormones. $J$ Neurosci. 2011;31(45):16107-16116.

75. Manber R, Baker FC, Gress JL. Sex differences in sleep and sleep disorders: a focus on women's sleep. Int J Sleep Disorders. 2006;1:7-15.

76. Roenneberg T, Kuehnle T, Juda M, et al. Epidemiology of the human circadian clock. Sleep Med Rev. 2007;11(6):429-438.

77. Cain SW, Dennison CF, Zeitzer JM, et al. Sex differences in phase angle of entrainment and melatonin amplitude in humans. J Biol Rhythms. 2010;25(4):288-296.

78. Van Reen E, Sharkey KM, Roane BM, et al. Sex of college students moderates associations among bedtime, time in bed, and circadian phase angle. J Biol Rhythms. 2013;28(6):425-431.

79. Wirz-Justice A, Wever RA, Aschoff J. Seasonality in freerunning circadian rhythms in man. Naturwissenschaften. 1984;71(6):316-319.

80. Manber R, Armitage R. Sex, steroids, and sleep: a review. Sleep. 1999;22(5):540-555.

81. Moline ML, Broch L, Zak R. Sleep in women across the life cycle from adulthood through menopause. Med Clin North Am. 2004;88(3):705-736.
82. Shibui K, Uchiyama M, Okawa M. Melatonin rhythms in delayed sleep phase syndrome. J Biol Rhythms. 1999;14(1):72-76.

83. Morris CJ, Aeschbach D, Scheer FA. Circadian system, sleep and endocrinology. Mol Cell Endocrinol. 2012;349(1):91-104.

84. NINDS Restless Legs Syndrome Information Page [webpage on the Internet]. Bethesda: National Institute of Neurological Disorders and Stroke; 2013; Available from: http://www.ninds.nih.gov/disorders/ restless_legs/restless_legs.htm. Accessed April 1, 2014.

85. Berger K, Luedemann J, Trenkwalder C, John U, Kessler C. Sex and the risk of restless legs syndrome in the general population. Arch Intern Med. 2004;164(2):196-202.

86. Manconi M, Govoni V, De Vito A, et al. Restless legs syndrome and pregnancy. Neurology. 2004;63(6):1065-1069.

87. Chong Y, Fryer CD, Gu Q. Prescription sleep aid use among adults: United States, 2005-2010. NCHS Data Brief. 2013;(127):1-8.

88. Kripke DF, Langer RD, Kline LE. Hypnotics' association with mortality or cancer: a matched cohort study. BMJ Open. 2012;2(1): e 000850 .

89. Krystal AD. Depression and insomnia in women. Clin Cornerstone. 2004;6 Suppl 1B:S19-S28.

90. Schutte-Rodin S, Broch L, Buysse D, Dorsey C, Sateia M. Clinical guideline for the evaluation and management of chronic insomnia in adults. J Clin Sleep Med. 2008;4(5):487-504.

91. Morin CM, Bootzin RR, Buysse DJ, Edinger JD, Espie CA, Lichstein KL. Psychological and behavioral treatment of insomnia:update of the recent evidence (1998-2004). Sleep. 2006;29(11):1398-1414.

92. Morin CM, Colecchi C, Stone J, Sood R, Brink D. Behavioral and pharmacological therapies for late-life insomnia: a randomized controlled trial. Jama. 1999;281(11):991-999.

93. Wu R, Bao J, Zhang C, Deng J, Long C. Comparison of sleep condition and sleep-related psychological activity after cognitive-behavior and pharmacological therapy for chronic insomnia. Psychother Psychosom. 2006;75(4):220-228.

94. Jacobs GD, Pace-Schott EF, Stickgold R, Otto MW. Cognitive behavior therapy and pharmacotherapy for insomnia: a randomized controlled trial and direct comparison. Arch Intern Med. 2004;164(17): 1888-1896.

95. Baker FC, Mitchell D, Driver HS. Oral contraceptives alter sleep and raise body temperature in young women. Pflugers Arch. 2001;442(5): 729-737.

96. Ye L, Pien GW, Ratcliffe SJ, Weaver TE. Gender differences in obstructive sleep apnea and treatment response to continuous positive airway pressure. J Clin Sleep Med. 2009;5(6):512-518.

97. How Is Sleep Apnea Treated? [webpage on the Internet]. Bethesda: National Heart, Lung, and Blood Institute; 2012; Available from: http:// www.nhlbi.nih.gov/health/health-topics/topics/sleepapnea/treatment. html. Accessed April 1, 2014.

98. Campos-Rodríguez F, Martínez-García MA, Montserrat JM. Gender differences in treatment recommendations for sleep apnea. Clinical Practice. 2012;9(5):565-578.

99. Shahar E, Redline S, Young T, et al. Hormone replacement therapy and sleep-disordered breathing. Am J Respir Crit Care Med. 2003; 167(9):1186-1192.

100. Manber R, Kuo TF, Cataldo N, Colrain IM. The effects of hormone replacement therapy on sleep-disordered breathing in postmenopausal women: a pilot study. Sleep 2003;26(2):163-168.

101. Veasey SC, Guilleminault C, Strohl KP, Sanders MH, Ballard RD, Magalang UJ. Medical therapy for obstructive sleep apnea: a review by the Medical Therapy for Obstructive Sleep Apnea Task Force of the Standards of Practice Committee of the American Academy of Sleep Medicine. Sleep. 2006;29(8):1036-1044.

102. Mundey K, Benloucif S, Harsanyi K, Dubocovich ML, Zee PC. Phase-dependent treatment of delayed sleep phase syndrome with melatonin. Sleep. 2005;28(10):1271-1278.

103. Cajochen C, Krauchi K, Danilenko KV, Wirz-Justice A. Evening administration of melatonin and bright light: interactions on the EEG during sleep and wakefulness. J Sleep Res. 1998;7(3):145-157. 
104. Paulus W, Dowling P, Rijsman R, Stiasny-Kolster K, Trenkwalder C, de Weerd A. Pathophysiological concepts of restless legs syndrome. Mov Disord. 2007;22(10):1451-1456.

105. Earley CJ, Allen RP, Beard JL, Connor JR. Insight into the pathophysiology of restless legs syndrome. J Neurosci Res. 2000; 62(5):623-628.

106. Winkelman JW. Considering the causes of RLS. Eur J Neurol. 2006; 13 Suppl 3:8-14.
107. Aurora RN, Kristo DA, Bista SR, et al. The treatment of restless legs syndrome and periodic limb movement disorder in adults-an update for 2012: practice parameters with an evidence-based systematic review and meta-analyses: an American Academy of Sleep Medicine Clinical Practice Guideline. Sleep. 2012;35(8):1039-1062.

108. Clavadetscher SC, Gugger M, Bassetti CL. Restless legs syndrome: clinical experience with long-term treatment. Sleep Med. 2004;5(5):495-500.

\section{Publish your work in this journal}

ChronoPhysiology and Therapy is an international, peer-reviewed, open access journal focusing on research into the cyclic variations and rhythmicity in physiological processes in the body and the research and development and optimal timing of administration of therapeutic targets to achieve improved outcomes and quality of life for the patient. The
Dovepress

manuscript management system is completely online and includes a very quick and fair peer-review system. Visit http://www.dovepress.com/ testimonials.php to read real quotes from published authors.

\footnotetext{
Submit your manuscript here: http://www.dovepress.com/chronophysiology-and-therapy-journal
} 